\title{
Comparison of Clinical Features and Personality Dimensions between Patients with Major Depressive Disorder and Normal Control
}

\author{
Ji-Won Hur ${ }^{1}$ \\ Yong-Ku Kim ${ }^{1,2}$ \\ 1Departments of Psychiatry and \\ 2Division of Brain Korea \\ 21 Biomedical Science \\ Korea University \\ College of Medicine, \\ Ansan Hospital, Ansan, Korea
}

\author{
Received May 5, 2009 \\ Revised July 3, 2009 \\ Accepted July 24, 2009 \\ Available online August 3, 2009

\section{Correspondence} \\ Yong-Ku Kim, MD, PhD \\ Department of Psychiatry, \\ Korea University \\ College of Medicine, \\ Ansan Hospital, 516 Gojan-dong, \\ Ansan 425-020, Korea \\ Tel $+82-31-412-5140$ \\ Fax +82-31-412-5144 \\ E-mail yongku@korea.ac.kr
}

\begin{abstract}
Objective Personality dimension is considered as a risk factor of depression. This study was to compare aggression, impulsivity, hopelessness, and TCI (temperament and character dimensions) between patients with major depressive disorder (MDD) and normal controls.

Methods A total of $56 \mathrm{MDD}$ patients and the same number of normal controls who were matched for age, gender, and education were recruited. All subjects completed the following questionnaires; Aggression Questionnaire (AQ), Beck Hopelessness Scale (BHS), Barratt Impulsiveness Scale, 11th Version (BIS-11), and Temperament and Character Inventory (TCI).

Results MDD patients were significantly higher scores in anger, hostility of AQ, BHS, motor impulsivity of BIS-11, and Harm Avoidances (HA) of TCI with all subscales of HA than normal controls, whereas novelty seeking 1 (NS1) (Exploratory of NS), Reward Dependence (RD) with RD3 (Attachment) - RD4 (Dependence), Self-Directedness (SD) with most subscales of SD, Cooperativeness (CO), and ST3 (Spiritual Acceptance) showed lower scores than normal controls. Moreover, BHS and HA, BIS and NS showed moderate positive correlation in MDD patients, while BHS and SD, HA and SD were negatively correlated.

Conclusion The present study showed unique clinical features, especially personality dimensions of patients with MDD. Our results could be applicable to suggest treatment process and to predict one's prognosis for depression in that psychological properties are important for drug compliance and treatment response.
\end{abstract}

KEY WORDS: Major depressive disorder, Temperament and Character Inventory, Aggression, Hopelessness, Impulsiveness.

Psychiatry Invest 2009;6:150-155

\section{Introduction}

Many psychological models of depression have been frequently discussed to indicate the structure and development of depression, although many traits appear to be unstable and easy to be changed by symptoms. ${ }^{1}$ Because traits and factors of mental disorder are important to decide on therapeutic approaches to the specific disorder, previous studies have tried to trace a variety of clinical features and personality dimensions.

Previous researches have narrowed down and focused on some clinical issues to investigate structure of depression for decades. Aggression has been proposed as one of possible psychological variables which explain the depression. ${ }^{2}$ Fava has also suggested that the $44 \%$ of the depressed outpatients reported having anger attacks. ${ }^{3}$ Besides, the relation between impulsivity and depression has been considered as a significant feature in depressed patients. ${ }^{4}$ There have been many studies that tried to explain the relation between impulsive aggression and depression with biological factor. ${ }^{5}$ Hopelessness has been considered an another essential factor related to de- 
pression, as a proximal sufficient cause of the symptoms of hopelessness depression. ${ }^{6}$ In other word, as hopeless individuals attribute their own negative life-event to stable, global, and internal courses, they could become depressed.

Personality dimension is also considered as a risk factor of depression. ${ }^{7}$ The studies on Temperament and Character Inventory (TCI) by Cloninger et $\mathrm{al}^{8}{ }^{8}$ have proposed the relation between personality and depression. TCI includes seven biosocial factors, comprised of four temperament dimensions and three character dimensions. The four dimensions of temperament are Novelty Seeking (NS), Harm Avoidance (HA), Reward Dependence (RD), and Persistence (P). Temperament is defined as stable and heritable dimensions, which is biologically and genetically determined early in life. These dimensions are known to correlate with dopaminergic, serotonic and noradrergic activity. The three dimensions of character are Self-Directedness (SD), Cooperativeness (CO), and SelfTranscendence (ST). Character means the second domain of personality which is predominantly determined by socialization processes during one's whole life. ${ }^{8}$ As TCI includes many variables which have many of implication and description respectively, various results about relationship with depression have been derived. ${ }^{9,10}$

Although previous studies have supported the correlation between some subscales of TCI and psychiatric disorders, ${ }^{11-14}$ there has been very a few study regarding personality dimensions and the correlation of clinical features and personality in major depressive disorder (MDD). Furthermore, some studies have limitations related to heterogeneity of demographic data, when the covariance such as age or education are not controlled appropriately. ${ }^{14}$

The aim of our study was to compare aggression, impulsivity, hopelessness and personality dimensions between MDD patients and normal controls while controlling for demographic variances; and to evaluate the correlations between variances. Our hypothesis was that MDD patients would have specific personality dimensions than normal control. We also assumed some relationship with the personality and clinical implications.

\section{Methods}

\section{Patricipants}

Among psychiatric patients newly admitted to the closed wards of the Department of Psychiatry, Korea University Medical Center Ansan Hospital, during January 2006 to January 2008, we recruited 56 major depression patients (22 males and 34 females) who met the Diagnostic and Statistical Manual (DSM-IV) criteria. ${ }^{15}$ All patients were medication-free for at least 4 months and had active sym- ptoms at the time of study enrollment. The psychopathological status of the patients was assessed by a trained physician the Hamilton Depression Rating Scale (HDRS). ${ }^{16}$ Patients with a history of any concomitant physical and psychiatric illness, such as anxiety disorder, substance or alcohol abuse, personality disorder and mental retardation were excluded. Patients were found to have a normal physical state as seen from normal values of blood and urine tests (SGOT, SGPT, hemoglobin, hematocrit, serum electrolytes, blood urea, and creatine). Patients were given a standard set of tests by the Venereal Disease Research Laboratory (VDRL) and had normal electrocardiogram (EKG) and electroencephalogram (EEG). For 2 years, through these inclusive/exclusive procedures, 56 MDD patients were selected among 74 MDD inpatients who performed the TCI and questionnaires.

A total of 56 normal control subjects (16 males and 40 females) were recruited. The normal controls were matched with the patients for gender, age and education. They were recruited through advertisement and screened using the Structured Clinical Interview for DSM-IV Axis I Disorders (SCID-I), non-patient version. They had neither medical/psychiatric illness nor family history of mental illness in first- and second-degree relatives. They received a modest fee for completing the interview.

Table 1 shows the demographic data of both groups. The subjects gave informed consent after the study protocol had been fully explained. This study was approved by the Institutional Review Board of Korea University Ansan Hospital.

\section{Measures}

All participants completed the following questionnaires. Aggression Questionnaire (AQ) is self-report measure which contains four subscales of aggression, including physical aggression, verbal aggression, anger, and hostility. ${ }^{17}$ Beck Hopelessness Scale (BHS) was designed to measure the construct of hopelessness that is related positively with depression. ${ }^{18}$ Barratt Impulsiveness Scale, 11th Version (BIS-11) is a short questionnaire developed to measure impulsiveness as a personality trait. ${ }^{19}$ All participants were also administered TCI, which is a selfreported 240 item forced-choice questionnaire. ${ }^{8}$ TCI measures temperament dimensions of NS, HA, RD, P, and character dimensions of SD, CO, ST.

\section{Statistical analysis}

Socio-demographic variables such as age and sex, and clinical history were compared between groups using independent t-tests and $\chi^{2}$ tests. Because there were only two groups, the t-test was performed to tap the differences in psychological measures between patients with 
TABLE 1. Demographic data for patients with major depressive disorder (MDD) and normal control subjects

\begin{tabular}{|c|c|c|c|c|}
\hline & Patients with MDD ( $N=56)$ & Normal control subjects $(\mathrm{N}=56)$ & $\dagger\left(x^{2}\right)$ & $\mathrm{p}$ \\
\hline Age (years) & $40.11 \pm 14.96$ & $37.32 \pm 3.98$ & -1.337 & 0.186 \\
\hline Education (years) & $11.21 \pm 3.04$ & $12.16 \pm 1.81$ & $1 / 88$ & 0.065 \\
\hline \multicolumn{5}{|l|}{ Sex } \\
\hline Male & 22 & 16 & $(1.434)$ & 0.231 \\
\hline Female & 34 & 40 & & \\
\hline Duration of illness (months) & $67.76 \pm 85.77$ & & & \\
\hline \multicolumn{5}{|l|}{ Total numbers of admissions } \\
\hline 0 time & $27(48.2 \%)$ & & & \\
\hline 1-3 time & $21(37.5 \%)$ & & & \\
\hline$\geq 4$ times & $8(14.3 \%)$ & & & \\
\hline Hamilton Depression Rating Scale scores & $24.2 \pm 3.3$ & & & \\
\hline
\end{tabular}

MDD and normal controls, also. All statistical analyses were done using Statistical Package for Social Science (SPSS) Version 12.0. The level of significance was $\mathrm{p}<$ 0.05 .

\section{Results}

\section{Comparison of the psychological measures}

The mean scores for each measure for both MDD group and normal control are presented in Table 2. MDD group were more like to experience anger (of AQ; 14.63 \pm 4.63 vs. 11.77 $\pm 3.50 ; \mathrm{t}=-3.34, \mathrm{p}=0.001$ ), hostility (of AQ; 19.40 \pm 6.64 vs. $15.20 \pm 5.89 ; \mathrm{t}=-3.07, \mathrm{p}<0.01)$, hopelessness (BHS 9.51 \pm 6.77 vs. $2.52 \pm 3.15 ; \mathrm{t}=-5.74, \mathrm{p}<0.001$ ), and impulsivity tendency (BIS total $51.57 \pm 9.95$ vs. $47.27 \pm$ 9.88; $\mathrm{t}=-2.02, \mathrm{p}<0.05$ ) including motor impulsivity (BIS; $15.51 \pm 4.27$ vs. $13.29 \pm 3.27 ; \mathrm{t}=-2.81, \mathrm{p}<0.01)$ than normal controls. MDD group also scored significantly higher on all subscales of HA, Worry, pessimism (HA1), Fear of uncertainty (HA2), Shyness (HA3), and Fatigability (HA4). Besides, Exploratory excitability (NS1), Two of RD subscales, Attachment (RD3) and Dependence (RD4), three of SD subscales, Responsibility (SD1), Purposefulness (SD2), Resourcefulness (SD3), and Enlightened second nature (SD5) were lower among MDD patients than controls. CO dimension (C) including Acceptance (C1), Empathy (C2), Helpfulness (C3), and Integrated conscience (C5) subscales and the subscale Spiritual acceptance (ST3) of ST were significantly lower in MDD patients.

\section{Correlations among scales in major depressive disorder patients}

In MDD patients, the correlation between AQ score and ST $(\mathrm{r}=0.338, \mathrm{p}<0.05)$, BHS score and HA $(\mathrm{r}=0.548$, $\mathrm{p}<0.01)$, BIS score and NS $(\mathrm{r}=0.518, \mathrm{p}<0.01)$, RD and $\mathrm{CO}(\mathrm{r}=0.431, \mathrm{p}<0.01), \mathrm{P}$ and $\mathrm{SD}(\mathrm{r}=0.270, \mathrm{p}<0.05), \mathrm{SD}$ and $\mathrm{CO}(\mathrm{r}=0.438, \mathrm{p}<0.01)$ were positive (Table 3$)$. Other variables show negative correlations; AQ total score and SD $(r=-0.372, p<0.05)$, AQ score and CO $(r=-0.394, p<$ $0.05)$, BHS score and SD ( $\mathrm{r}=-0.598, \mathrm{p}<0.01)$, BHS score and $\mathrm{CO}(\mathrm{r}=-0.336, \mathrm{p}<0.05)$, BIS score and $\mathrm{P}(\mathrm{r}=-0.364$, $\mathrm{p}<0.05)$, HA and SD $(\mathrm{r}=-0.555, \mathrm{p}<0.01)$, and HA and $\mathrm{CO}(\mathrm{r}=-0.450, \mathrm{p}<0.01)$.

\section{Discussion}

The aim of the present study was to explore the psychological properties of MDD compared with normal controls and to tap the risk factor or vulnerability on clinical status and personality dimensions. The major implications of our study are as follows.

The first major finding of this study is that anger, hostility, hopelessness, and impulsivity including motor impulsivity were significant clinical traits in MDD as previous authors have described; Anger and hostility, which were two of subscales of AQ, were higher in MDD patients than normal control (NC) subjects. Pasquini and his colleagues commented also that anger and hostility as well as irritability and aggressiveness are frequent present in depressive patients. ${ }^{20}$ In addition, as previous studied suggested the relationship of hopelessness and depression, similar results was presented in this study also. ${ }^{6,21}$ One of the most interesting thing in our study is probably higher impulsivity including motor impulsivity in MDD patients than NC. In fact, Fava et al. ${ }^{22}$ mentioned important issues concerning the impact of MDD on violent and aggressive behavior. Also, there was a notable opinion that activation dimension in depression disorders deserves greater clinical recognition and research. ${ }^{23}$ Similarly, Arciero and Guidano observed prominent symptoms of activation which might be related to depressed patient's cognitive and emotional factors. ${ }^{24}$ Considering these points, it is understandable that MDD patients have rather activat- 
ion factor, impulsivity including motor impulsivity than NC.

The second major finding of this study is that MDD patients showed higher harm-avoidance, lower RD, SD, and $\mathrm{CO}$ in TCI compared to normal controls. These re- sults concur with previous studies that reported positive relation with $\mathrm{HA}$ and negative correlation with $\mathrm{SD}, \mathrm{CO}$ in depressed groups. ${ }^{9,11,13,25} \mathrm{HA}$ is the result of depression, and may reflect neurobiological changes in the de-

TABLE 2. Comparison of clinical features and personality dimensions between patients with major depressive disorder (MDD) and normal control subjects

\begin{tabular}{|c|c|c|c|c|}
\hline & Patients with MDD ( $N=56)$ & Normal control $(\mathrm{N}=56)$ & $\dagger$ & $\mathrm{p}$ \\
\hline \multicolumn{5}{|l|}{ Clinical features } \\
\hline Aggression questionnaire & $62.97 \pm 18.35$ & $56.96 \pm 17.60$ & -1.558 & 0.123 \\
\hline Physical aggression & $18.57 \pm 7.38$ & $17.52 \pm 5.38$ & -0.786 & 0.434 \\
\hline Verbal aggression & $10.37 \pm 3.66$ & $10.88 \pm 3.15$ & 0.697 & 0.488 \\
\hline Anger & $14.63 \pm 4.63$ & $11.77 \pm 3.50$ & -3.342 & 0.001 \\
\hline Hostility & $19.40 \pm 6.64$ & $15.20 \pm 5.89$ & -3.066 & 0.003 \\
\hline Beck Hopelessness Scale & $9.51 \pm 6.77$ & $2.52 \pm 3.15$ & -5.738 & $<0.001$ \\
\hline Barratt Impulsiveness Scale & $51.57 \pm 9.95$ & $47.27 \pm 9.88$ & -2.017 & 0.047 \\
\hline Nonplanning impulsiveness & $20.40 \pm 5.77$ & $18.70 \pm 3.96$ & -1.543 & 0.131 \\
\hline Motor impulsiveness & $15.51 \pm 4.27$ & $13.29 \pm 3.27$ & -2.805 & 0.006 \\
\hline Cognitive impulsiveness & $15.66 \pm 3.07$ & $18.46 \pm 17.24$ & 0.952 & 0.344 \\
\hline \multicolumn{5}{|l|}{ Temperament and Character Inventory } \\
\hline Novelty Seeking (NS) & $15.89 \pm 5.69$ & $16.20 \pm 5.98$ & 0.276 & 0.783 \\
\hline Exploaratory excitability (NS1) & $4.38 \pm 2.20$ & $5.18 \pm 2.07$ & 1.988 & 0.049 \\
\hline Impulsiveness (NS2) & $3.86 \pm 1.94$ & $3.73 \pm 2.24$ & -0.315 & 0.753 \\
\hline Extravagance (NS3) & $3.57 \pm 1.90$ & $3.89 \pm 2.08$ & 0.855 & 0.394 \\
\hline Disorderliness (NS4) & $4.00 \pm 2.13$ & $3.39 \pm 2.04$ & -1.535 & 0.128 \\
\hline Harm Avoidance (HA) & $23.20 \pm 6.99$ & $17.45 \pm 6.09$ & -4.643 & $<0.001$ \\
\hline Anticipatory worry (HAl) & $6.16 \pm 2.74$ & $3.89 \pm 2.41$ & -4.655 & $<0.001$ \\
\hline Fear of uncertainty (HA2) & $5.05 \pm 1.46$ & $4.13 \pm 1.60$ & -3.214 & 0.002 \\
\hline Attachment (HA3) & $4.98 \pm 2.01$ & $3.91 \pm 2.30$ & -2.610 & 0.010 \\
\hline Dependence (HA4) & $6.96 \pm 2.43$ & $5.52 \pm 1.79$ & -3.590 & 0.001 \\
\hline Reward Dependence (RD) & $14.14 \pm 3.54$ & $16.25 \pm 2.86$ & 3.468 & 0.001 \\
\hline Sentimentality (RDI) & $6.96 \pm 1.91$ & $7.11 \pm 1.81$ & 0.407 & 0.685 \\
\hline Attachment (RD3) & $4.36 \pm 2.09$ & $5.52 \pm 1.88$ & 3.089 & 0.003 \\
\hline Dependence (RD4) & $2.82 \pm 1.34$ & $3.79 \pm 1.73$ & 3.296 & 0.001 \\
\hline Persistence (P) & $4.58 \pm 3.54$ & $16.25 \pm 2.86$ & 0.143 & 0.886 \\
\hline Self-Directedness (SD) & $22.44 \pm 7.94$ & $27.79 \pm 9.04$ & 3.310 & 0.001 \\
\hline Responsibility (SDI) & $4.89 \pm 2.28$ & $6.11 \pm 1.66$ & 3.225 & 0.002 \\
\hline Purposefulness (SD2) & $3.79 \pm 1.96$ & $5.14 \pm 1.71$ & 3.904 & $<0.001$ \\
\hline Resourcefulness (SD3) & $2.18 \pm 1.44$ & $2.77 \pm 1.43$ & 2.174 & 0.032 \\
\hline Self-acceptance (SD4) & $5.35 \pm 2.61$ & $5.84 \pm 2.76$ & 0.969 & 0.335 \\
\hline Congruent second nature (SD5) & $6.45 \pm 2.40$ & $8.66 \pm 2.28$ & 4.999 & $<0.001$ \\
\hline Cooperativeness (C) & $27.91 \pm 6.17$ & $32.29 \pm 5.11$ & 4.295 & $<0.001$ \\
\hline Acceptance $(\mathrm{Cl})$ & $5.45 \pm 1.78$ & $6.27 \pm 1.68$ & 2.514 & 0.013 \\
\hline Empathy (C2) & $4.14 \pm 1.46$ & $5.04 \pm 1.36$ & 3.350 & 0.001 \\
\hline Helpfulness (C3) & $5.25 \pm 1.86$ & $6.64 \pm 2.08$ & 3.739 & $<0.001$ \\
\hline Compassion vs. revenge (C4) & $7.32 \pm 2.38$ & $7.66 \pm 2.31$ & 0.766 & 0.446 \\
\hline Intergrated conscience (C5) & $5.75 \pm 1.34$ & $6.80 \pm 1.26$ & 4.295 & $<0.001$ \\
\hline Self-Transcendence (ST) & $12.64 \pm 6.27$ & $13.32 \pm 6.20$ & -0.576 & 0.566 \\
\hline Self-forgetfulness (STI) & $4.88 \pm 2.57$ & $4.34 \pm 2.26$ & -1.173 & 0.243 \\
\hline Transpersonal identification (ST2) & $3.14 \pm 1.82$ & $3.27 \pm 1.90$ & 0.355 & 0.723 \\
\hline Spiritual acceptance (ST3) & $4.63 \pm 3.09$ & $5.89 \pm 3.51$ & 2.029 & 0.045 \\
\hline
\end{tabular}


TABLE 3. Correlations among clinical features and personality dimensions within the Major depressive disorder group

\begin{tabular}{|c|c|c|c|c|c|c|c|c|c|}
\hline & $A Q$ & BHS & BIS & NS & $\mathrm{HA}$ & RD & $\mathrm{P}$ & SD & $\mathrm{CO}$ \\
\hline BHS & 0.240 & & & & & & & & \\
\hline BIS & -0.049 & -0.001 & & & & & & & \\
\hline NS & 0.133 & -0.116 & $0.518^{* *}$ & & & & & & \\
\hline HA & 0.280 & $0.548^{* *}$ & -0.029 & -0.209 & & & & & \\
\hline RD & -0.248 & -0.201 & -0.079 & -0.036 & -0.154 & & & & \\
\hline$P$ & 0.049 & -0.175 & $-0.364^{*}$ & -0.251 & -0.129 & -0.161 & & & \\
\hline SD & $-0.372^{*}$ & $-0.598^{* *}$ & -0.122 & -0.152 & $-0.555^{* *}$ & 0.162 & $0.270^{*}$ & & \\
\hline $\mathrm{CO}$ & $-0.394^{*}$ & $-0.336^{*}$ & 0.034 & -0.074 & $-0.450^{* *}$ & $0.431^{* *}$ & 0.134 & $0.438^{* *}$ & \\
\hline ST & $0.338^{*}$ & 0.112 & -0.091 & -0.071 & -0.178 & -0.007 & 0.261 & -0.224 & 0.044 \\
\hline
\end{tabular}

${ }^{*} \mathrm{p}<0.05,{ }^{* *} \mathrm{p}<0.01$. AQ: Aggression Questionnaire, BHS: Beck Hopelessness Scale, BIS: Barratt Impulsiveness Scale, NS: Novelty Seeking, HA: Harm Avoidance, RD: Reward Dependence, P: Persistence, SD: Self-Directedness, CO: Cooperativeness, ST: Self-Transcendence

pressive patients. ${ }^{7}$ Moreover, considering that high score on HA may be better interpreted as reflecting a state rather than a trait, high HA in the "inpatients with MDD" could be understood. On the other hand, MDD patients scored significantly lower RD than normal controls. RD could influence over resilience against psychosocial stressors which results in increased cortisol secretion, subordinance, depressive or suicidal ideation and low selfesteem. ${ }^{12}$ Besides, tendency of $\mathrm{SD}$, which is correlated with accepting responsibility, long-term goals and selfesteem, ${ }^{26}$ was lower in depressed patients than normal controls. Above features of SD could be useful reasons to explain personality of MDD patients in regard to their feelings of futility about future and low self-esteem. Also, as above mentioned, lower score on $\mathrm{CO}$ was founded in MDD groups as previous researcher pointed out. ${ }^{27}$ These findings might indicate malfunctioning occurrence pattern. Some authors have proposed yet that $\mathrm{CO}$ as well as $\mathrm{SD}$ is a dimension which reflects a general psychopathology. ${ }^{9}$ Focusing the concept of CO-socially intolerant, unhelpful, and revengeful-, it seems that this personality dimension makes patient's social adjustment be difficult

In our study, MDD patients showed significant low exploratory excitability score among NS subscales, also. Even though many researchers pointed insignificant score on subscales NS, ${ }^{28,29}$ Hansenne et al. ${ }^{9}$ have reported the results same as ours, interestingly. They explained that the result could be observed in that low exploratory excitability means not doing initiative novel behaviors and producing active exploration of the environment. Finally, spiritual acceptance of ST, which was identified as being important to older adult's sense of well-being, ${ }^{30}$ is lower in MDD group than normal. In the view of biological model for this illness, this result is reasonable. Since correlation between spiritual acceptance and 5HT1A genotype was defined ${ }^{31}$ which response in unipolar depression. ${ }^{32}$

First, BHS (hopelessness) and HA, BIS (Impulsivity) and NS showed moderate positive Pearson correlation coefficients in MDD patients. The former results is similar with a previous study that suggested the biological effects of relationship between hopelessness and avoidance behavior. ${ }^{12}$ In the other hand, although we didn't describe about correlations in normal controls above, the same results with latter one was found among normal control in case of BIS and NS $(r=0.586, p<0.01)$. Hence, it is considered this relationship about BIS and NS is not due to the characteristic trait of depression.

Secondly, BHS (hopelessness) and SD, HA and SD were negatively correlated. Cloninger et al. ${ }^{8}$ have suggested individual with low level in SD to be weak, fragile, ineffective, irresponsible, and poorly integrated when they are not performing to the direction of a mature leader. These features could indicate negative correlations between SD and hopelessness, HA. In addition, Chien and Dunner $^{29}$ and other researchers ${ }^{25}$ have described that reductions in depressive symptoms was accompanied by decreases in HA and increases in SD longitudinally same as our results.

In this study, we explored the clinical features, temperament and characteristics of patients with MDD as a cue factor. Sato and Uehara have asserted the study of personality as a precursor of depression helped us to understand treatment and etiology. ${ }^{33}$ Since most studies didn't cover symptoms of 'activation' such as anger, irritability, aggressiveness, and hostility among depressed patients, ${ }^{23}$ our results about not only TCI but also AQ, BIS and BHS could be a references for further studies. Another strong point of our study is that, when the analysis was administered, statistical traits such as sex and education which could carry unwanted effects were controlled for homogeneity of demographic data.

Our study is limited by sampling and cross-sectional design. Since only inpatients with MDD but not outpatient were sampled, there might be bias by severity of illness. Besides, we didn't perform longitudinal study 
for following the phase of above psychological properties after discharge. Finally, we didn't classified as either endogenic or exogenic depression which have different processes of onset, procedure and prognosis. Further studies are necessary to conclude that these results could be maintained with refined and classified patients in the various courses of depression.

Our results suggest that MDD patients have significant clinical features and personality dimensions, which were related partly. The results included activation dimensions as anger, hostility and impulsivity as well as personality dimensions. Further longitudinal study is necessary to confirm whether the characteristics of MDD patients are state or trait.

\section{- Acknowledgments}

This work was supported by a grant from Korean Society of Depression and Bipolar disorders.

\section{REFERENCES}

1. Peselow ED, Sanfilipo MP, Fieve RR. Relationship between hypomania and personality disorders before and after successful treatment. Am J Psychiatry 1995;152:232-238.

2. Van Praag HM. Depression, aggression, and anxiety: a biological hypothesis about their interrelation. Eur Neuropsychopharmacol 1992;2: $393-402$.

3. Fava M, Rosenbaum JF, Pava JA, McCarthy MK, Steingard RJ, Bouffides E. Anger attacks in unipolar depression, Part 1: clinical correlates and response to fluoxetine treatment. Am J Psychiatry 1993; 150:1158-1163.

4. Lejoyeux M, Arbaretaz M, McLoughlin M, Ades J. Impulse control disorders and depression. J Nerv Ment Dis 2002;190:310-314.

5. New AS, Gelernter J, Goodman M, Mitropoulou V, Koenigsberg H, Silverman J, et al. Suicide, impulsive aggression, and HTR1B genotype. Biol Psychiatry 2001;50:62-65.

6. Abramson LY, Metalsky GI, Alloy LB. Hopelessness depression: a theory-based subtype of depression. Psychol Rev 1989;96:358-372.

7. Naito M, Kijima N, Kitamura T. Temperament and character inventory (TCI) as predictors of depression among Japanese college students. J Clin Psychol 2000;56:1579-1585.

8. Cloninger CR, Przybeck TR, Svrakic DM, Wetzel RD. The temperament and character inventory (TCI): a guide to its development and use: center for psychobiology of personality, Washington University, St. Louis, Mo; 1994.

9. Hansenne M, Reggers J, Pinto E, Kjiri K, Ajamier A, Ansseau M. Temperament and character inventory (TCI) and depression. J Psychiatr Res 1999;33:31-36.

10. Peirson AR, Heuchert JW. The relationship between personality and mood: comparison of the BDI and the TCI. Personality and Individual Differences 2001;30:391-399.

11. Kusunoki K, Sato T, Taga C, Yoshida T, Komori K, Narita T, et al. Low novelty-seeking differentiates obsessive-compulsive disorder from major depression. Acta Psychiatr Scand 2000;101:403-405.

12. van Heeringen C, Audenaert K, Van Laere K, Dumont F, Slegers G, Mertens J, et al. Prefrontal 5-HT2a receptor binding index, hopelessness and personality characteristics in attempted suicide. J Affect Disord 2003;74:149-158.
13. Marijnissen G, Tuinier S, Sijben AES, Verhoeven WMA. The temperament and character inventory in major depression. J Affect Disord 2002;70:219-223.

14. Loftus ST, Garno JL, Jaeger J, Malhotra AK. Temperament and character dimensions in bipolar I disorder: a comparison to healthy controls. J Psychiatr Res 2008;42:1131-1136.

15. American Psychiatric Association. Diagnostic and statistical manual of mental disorders, 4rd Ed, Washington, DC: American Psychiatric Press; 1994.

16. Hamilton M. A rating scale for depression. J Neurol Neurosurg Psychiatry 1960;23:56-62.

17. Harris JA. A further evaluation of the aggression questionnaire: issues of validity and reliability. Behav Res Ther 1997;35:1047-1053.

18. Beck AT, Weissman A, Lester D, Trexler L. The measurement of pessimism: the hopelessness Scale. J Consult Clin Psychol 1974;42:861865.

19. Patton JH, Stanford MS, Barratt ES. Factor structure of the Barratt impulsiveness scale. J Clin Psychol 1995;51:768-774.

20. Pasquini M, Picardi A, Biondi M, Gaetano P, Morosini P. Relevance of anger and irritability in outpatients with major depressive disorder. Psychopathology 2004;37:155-160.

21. Henkel V, Bussfeld P, Moller HJ, Hegerl U. Cognitive-behavioural theories of helplessness/hopelessness: valid models of depression? Eur Arch Psychiatry Clin Neurosci 2002;252:240-249.

22. Fava M, Nolan S, Kradin R, Rosenbaum J. Gender differences in hostility among depressed and medical outpatients. J Nerv Ment Dis 1995; 183:10-14.

23. Biondi M, Picardi A, Pasquini M, Gaetano P, Pancheri P. Dimensional psychopathology of depression: detection of an 'activation' dimension in unipolar depressed outpatients. J Affect Disord 2005;84:133-139.

24. Arciero G, Guidano VF. Experience, explanation, and the quest for coherence. Washington, DC: American Psychological Association; 2000

25. Hirano S, Sato T, Narita T, Kusunoki K, Ozaki N, Kimura S, et al. Evaluating the state dependency of the temperament and character inventory dimensions in patients with major depression: a methodological contribution. J Affect Disord 2002;69:31-38.

26. Svrakic DM, Draganic S, Hill K, Bayon C, Przybeck TR, Cloninger $\mathrm{CR}$, et al. Temperament, character, and personality disorders: etiologic, diagnostic, treatment issues. Acta Psychiatr Scand 2002;106:189-195.

27. Kimura S, Sato T, Takahashi T, Narita T, Hirano S, Goto M. Typus melancholicus and the temperament and character inventory personality dimensions in patients with major depression. Psychiatry Clin Neurosci 2000;54:181-189.

28. Brown SL, Svrakic DM, Przybeck TR, Cloninger CR. The relationship of personality to mood and anxiety states: a dimensional approach. J Psychiatr Res 1992;26:197-211.

29. Chien AJ, Dunner DL. The tridimensional personality questionnaire in depression: state versus trait issues. J Psychiatr Res 1996;30:21-27.

30. Reed PG. Self-transcendence and mental health in oldest-old adults. Nurs Res 1991;40:5-11.

31. Lorenzi C, Serretti A, Mandelli L, Tubazio V, Ploia C, Smeraldi E, et al. 5-HT 1A polymorphism and self-transcendence in mood disorders. Am J Med Genet B Neuropsychiatr Genet 2005;137B:33-35.

32. Lesch KP, Mayer S, Disselkamp-Tietze J, Hoh A, Wiesmann M, Osterheider M, et al. 5-HT1A receptor responsivity in unipolar depression. Evaluation of ipsapirone-induced ACTH and cortisol secretion in patients and controls. Biol Psychiatry 1990;28:620-628.

33. Sato T, Uehara T. Yokuutsu to jinkaku [Depression and personality]. Archives of Psychiatric Diagnostics and Clinical Evaluation 1995;6: $399-428$. 